\title{
La consolidación de competencias en la formación de gestores culturales: preparación práctica para la gestión, coordinación y montaje de eventos expositivos.
}

\author{
Nuria Ramón ${ }^{\mathrm{a}}$-Marqués Ana Martí-Tesón ${ }^{\mathrm{b}}$, Joan Aliaga-Morell ${ }^{\mathrm{c}}$ \\ ${ }^{a b c}$ Departamento de Comunicación Audiovisual, Documentación e Historia del Arte, UPV a nramon@har.upv.es. \\ banmartes@upv.es, ${ }^{\mathrm{b}}$ jaliaga@upv.es
}

\begin{abstract}
Cultural Management is a profession that has been consolidated in the last twenty years. The training of professionals in this field had been carried out from individual practice and self-taught training. Currently we have the opportunity to train future professionals with a regulated, quality and practical training. Our challenge is to be able to provide all the appropriate theoretical content and put it into practice in a specific case that brings us closer to the future professional reality. In this article we comment on the experience that a group of teachers from the master's course "Management Methods in Institutions and Cultural Spaces" have developed to coordinate and develop an exhibition at the Centro del Carmen in Valencia, on the art collection of the Polytechnic University of València on the occasion of the 50th anniversary of the institution, as a practice in which we have worked applying face-to-face classes and online activities with different effective methodologies, among which we highlight the Problem-Based Learning method, ServiceLearning and Cooperative Learning method.
\end{abstract}

Keywords: cultural management, exhibition management, cooperative learning, Service Learning, UPV artistic funds, temporary exhibition, transversal learning.

\section{Resumen}

La Gestión Cultural es una profesión que se ha ido consolidando en los últimos veinte años. La formación de profesionales en este ámbito se venía realizando desde la práctica individual y la formación autodidacta. En la actualidad tenemos la oportunidad de formar a los futuros profesionales con una formación reglada, de calidad y práctica. Nuestro reto consiste en lograr aportar todos los contenidos teóricos adecuados y ponerlos en práctica en un caso específico que nos aproxime a la futura realidad profesional. En este articulo comentamos la experiencia que hemos desarrollado un grupo de docentes de la asignatura de máster "Métodos de Gestión en Instituciones y Espacios Culturales" para coordinar y desarrollar una exposición en el Centro del Carmen de Valencia sobre la colección de arte de la Universitat Politècnica de València con motivo del 50 aniversario de la institución, como una práctica en la que hemos trabajado aplicando clases presenciales y actividades online con distintas metodologías que han resultado efectivas entre las que destacamos el método de Aprendizaje Basado en Problemas, el Aprendizaje - Servicio y el método del Aprendizaje Cooperativo.

Palabras clave: gestión cultural, gestión de exposiciones, aprendizaje cooperativo, AprendizajeServicio, fondos artísticos UPV, exposición temporal, aprendizaje transversal. 


\section{Introducción}

La Gestión Culturales es una profesión que, aunque apareció en los años 80 del siglo XX, en el contexto español, no se ha impartido en la enseñanza reglada hasta hace cerca de 10 años (Cabañés, 2017). Se trata de una profesión poco visibilizada en la que nos enfrentamos al reto de lograr una formación integral, pero ampliamente multidisciplinar que abarque desde la administración de empresas a la dirección de recursos humanos, la gestión de espacios y eventos, el marketing cultural, y un exhaustivo conocimiento del elemento del patrimonio cultural y la sociedad actual (La Asociación Española de Gestores de Patrimonio Cultural , s.f.).

En los últimos años, sólo la Universidad de Huelva oferta el Grado de Gestión Cultural como tal, sin embargo, por lo que respecta a los estudios de posgrado, son numerosas las universidades tanto dentro del ámbito nacional como internacional las que proporcionan la posibilidad de cursar un máster especializado en dicha disciplina. Este hecho contribuye a que, en el máster perteneciente a nuestra asignatura, los perfiles profesionales de nuestro alumnado sean diverso pudiéndose matricular en este alumnos/as que se han formado en historia del arte, bellas artes, arqueología, danza, música o algunos/as, e incluso procedentes de carreras más variadas como periodismo, informática o incluso ingenierías. Por tanto, se trata de perfiles muy variados, una cualidad que intentamos aprovechar en su formación interdisciplinar.

Por otro lado, existen pocas publicaciones docentes que versen sobre el entorno de la docencia de la gestión cultural, con lo que esta publicación pretende suponer un avance en este aspecto, remarcando los aspectos positivos de las experiencias junto a las dificultades encontradas.

Uno de los trabajos más importantes de la gestión cultural consiste en la organización de eventos y, dentro de este campo es fundamental la gestión de eventos expositivos. Algunos artículos han corroborado la importancia de disponer de estudios especializados en gestión cultural o el comisariado de exposiciones, para lograr una salida profesional, pues más del $50 \%$ de los empleados de museos en Estados Unidos, ostentan un título de estas características (Schonfeld, Westermann, 2015). Asimismo, diversas fuentes inciden en la importancia de realizar prácticas que aporten experiencia al alumnado de este tipo de estudios (Sullivan, s.f.; Krzys Acord, 2010; Cochran, 2017), pero como cometamos, la mayoría de ellas están escritas en inglés y apenas hay investigaciones en el ámbito nacional.

En el Máster Interuniversitario de Gestión Cultural que se imparte conjuntamente entre la Universitat Politècnica de Valencia y la Universitat de València se incluye un módulo bajo el título Métodos de Gestión en Instituciones y Espacios Culturales. En este módulo participan un total de tres asignaturas específicas enfocadas a proporcionar al alumno/a las herramientas necesarias para llevar a cabo las tareas propias de gestión de eventos y, de una forma más concreta, en el campo de las exposiciones artísticas temporales. En este sentido, el equipo docente generó un acto de evaluación que consistía en la gestión, coordinación y montaje de una exposición sobre los fondos artísticos de la UPV aprovechando la celebración del 50 aniversario de nuestra institución. Todo ello, favoreció a la imbricación del profesorado y de las asignaturas de manera transversal.

El presente proyecto se planteó como una actividad dentro de la asignatura Métodos de Gestión en Instituciones y Espacios Culturales perteneciente al Máster Universitario en Gestión Cultural, ofertado por la Universitat de València-Estudi General y la Universitat Politècnica de València, en colaboración con el Centre del Carme Cultura Contemporània. Se trata de una asignatura optativa de 10 créditos ECTS, y en el curso 2018-2019 contamos con 35 alumnos procedentes de diferentes disciplinas y de edades muy variadas (entre 22 y 45 años), de origen internacional, incluyendo países como Alemania, Italia, China o Argentina. 
El día de la presentación de la asignatura esta práctica se explicó a los estudiantes como una actividad práctica de gestión y coordinación que se iba a materializar en una exposición temporal, en un principio en el mes de mayo, aprovechando la finalización de la asignatura. Sin embargo, debido a modificaciones en la agenda cultural de la sala de exposiciones la inauguración de la muestra se tuvo que trasladar al mes de septiembre de 2019, debido a que el Centre del Carme Cultura Contemporània es uno de los centros culturales más activos de la ciudad de Valencia. Las obras que se iban a utilizar para dicha muestra formaban parte del Fondo de Arte de la UPV que, además de aprovechar para darle difusión al resto de la sociedad valenciana, nos permitió sumar esta iniciativa a los actos conmemorativos del 50 aniversario de nuestra universidad.

Según la bibliografía docente relacionada con el tema del montaje de exposiciones y el comisariado, existen diversos modos de aproximarse a la práctica, y en nuestro caso nos hemos inspirado en el modelo propuesto por Pat Villeneuve (2019) en el que defiende la puesta en práctica de la nueva museología, con el museo centrado en el visitante, fomentando la capacidad educativa y el museo participativo, defendiendo la postura de un equipo curatorial colaborativo.

El motivo primordial de la actividad que desarrollaron los alumnos y las alumnas consistió en poner en práctica los conocimientos teóricos adquiridos en las tres asignaturas que conformaban el bloque: Gestión de Museos y exposiciones, Gestión de Patrimonio Documental y Cinematográfico y Gestión y Organización de Eventos Culturales, esta última fue la asignatura que se encargó de la coordinación entre las tres. Además, con este ejercicio se pretendía subsanar la necesidad de la práctica de manera que los estudiantes pudieran enfrentarse a supuestos reales. Para ello se determinó utilizar la metodología del aprendizaje basado en la resolución de problemas, al tener que afrontar la dificultad de montar una exposición con los medios de que disponíamos, y los problemas derivados, a partir de situaciones de la vida real (Barrows, 1986). También aplicamos la metodología del Aprendizaje - Servicio al relacionar la práctica de la exposición con la misma universidad en la que estudian los alumnos/as y la celebración de los primeros 50 años de la institución (Francisco y Moliner, 2010; Martínez-Odría, 2007). Igualmente, utilizamos el método del Aprendizaje Cooperativo al trabajar en grupos grandes con distintas tareas que dependían unos de otros, facilitando la interdependencia positiva (Johnson, R. y Johnson D.W., 1994) (Díaz, 2005).

Con ello, los docentes de las asignaturas pretendíamos que nuestros alumnos/as pusieran en práctica la teoría trabajada en clase acerca de la gestión cultural, en general y de espacios expositivos en particular, con la necesidad de trabajar en equipo, con los medios y recursos más adecuados y comprobar en la práctica los problemas e incidencias que podían surgir. Es importante incidir en el hecho de que este trabajo formó parte de una estrategia fundamental para ofrecer al alumnado la calidad en la docencia que implicó de manera transversal a otras materias que se impartían en el mismo máster como: la profesión del gestor cultural y las políticas culturales, gestión del conocimiento y recursos informáticos y el patrimonio como recurso económico sostenible, el marketing, planificación cultural e incluso legislación o marco jurídico aplicado a la protección del patrimonio y a los museos. En definitiva, teníamos la oportunidad de poner en práctica gran parte de los contenidos estudiados en distintas materias del máster.

Tal y como hemos indicado anteriormente, a lo largo del curso 2019-2020, la Universitat Politècnica de València celebró su 50 aniversario. El impacto del evento y la proximidad académica con los alumnos convertía este acontecimiento en una oportunidad para elegir la colección del Fondo de Arte de la UPV como tema de la actividad. Al ser un organismo de la propia Universidad, los profesores considerábamos que el personal encargado de gestionar el Fondo estaba más sensibilizado en aspectos como la transmisión de conocimiento y aprendizaje de los estudiantes que era, en definitiva, el objetivo primordial 
de este ejercicio; poner a disposición de los estudiantes todo el engranaje de la propia universidad y acercarnos a la metodología del aprendizaje servicio.

Además, el poder realizar una exposición de la colección de la UPV permitía difundir desde el centro de la ciudad de Valencia, en un espacio prestigioso, el patrimonio artístico que la institución conserva, fomentando la proyección de Universidad en la difusión cultural de la sociedad, que era uno de los principios hacia los que se encaminaba la UPV con el objetivo 2020.

\section{Objetivos}

El principal objetivo que se pretendía generar con esta práctica era que el alumno/a pudiera enfrentarse a un supuesto real a partir de la puesta en práctica en grupo de los conocimientos teóricos que habían ido adquiriendo a lo largo de las asignaturas del Máster, y, más concretamente en la organización de eventos. Este objetivo permitía alcanzar el desarrollo de una serie de habilidades fundamentales para ejercer la actividad de gestor de exposiciones.

A este objetivo general, se le añadían otros objetivos específicos que se resumían en:

- Desarrollar la capacidad por parte del estudiante para relacionarse con el personal especializado que conforma el Fondo de Arte (dirección, documentalista, informático) para lo que era fundamental aplicar las habilidades aprendidas en clase con el fin de trabajar con equipos humanos multidisciplinares.

- Aprender las competencias relacionadas con la práctica de exponer y redactar un proyecto de gestión cultural desde un punto de vista profesional adaptado al Centre del Carme Cultura Contemporània. Para lograr este objetivo específico era necesaria la investigación del espacio para conocer la institución y relacionándose con los profesionales que la integran.

- Aprender a programar, gestionar y difundir una muestra expositiva con auténticas obras de arte de autores renombrados.

- Utilizar los recursos adecuados para trabajar en equipo con un grupo de más de 40 personas, de modo que se pueda interactuar entre los grupos y se coordine un evento conjunto.

- Utilizar en la práctica los principios de la nueva museografía, crítica y participativa.

\section{Desarrollo de la innovación}

Tal y como hemos indicado anteriormente, nuestra actividad consistía en la coordinación, gestión y organización de una exposición temporal dentro de una asignatura de máster de 10 créditos ETC's, distribuidos a lo largo del segundo semestre del curso 2018/19.

Para poder comprender la envergadura de este trabajo, debemos tener en cuenta que un evento de estas características implica diferentes departamentos que debían de realizar las siguientes acciones:

Protocolo:

- Comunicarse con las instituciones para difundir el evento.

- Comunicación con la prensa.

- Diseñar las invitaciones.

- Búsqueda de patrocinadores.

- Organización y coordinación de la inauguración de la exposición.



Congreso In-Red (2020) 
Comunicación:

- Desarrollar la página Web del evento.

- Gestión y mantenimiento de las Redes Sociales.

Gestión de Obras:

- Gestión de Préstamos, seguros y traslados de las obras que iban a ser expuestas.

Diseño:

- Desarrollo del proyecto expositivo.

- Montaje de la exposición a partir del uso de herramientas 3D.

- Gráfica de la exposición.

Catálogo:

- Diseño y edición del catálogo de la exposición.

- Maquetación del catálogo con la herramienta In Desing.

- Permisos y gestión de las fotografías de las obras.

- Gestión y producción de los textos y fichas del catálogo.

Didáctica:

- Visitas guiadas.

- Talleres y actividades paralelas.

- Conferencias

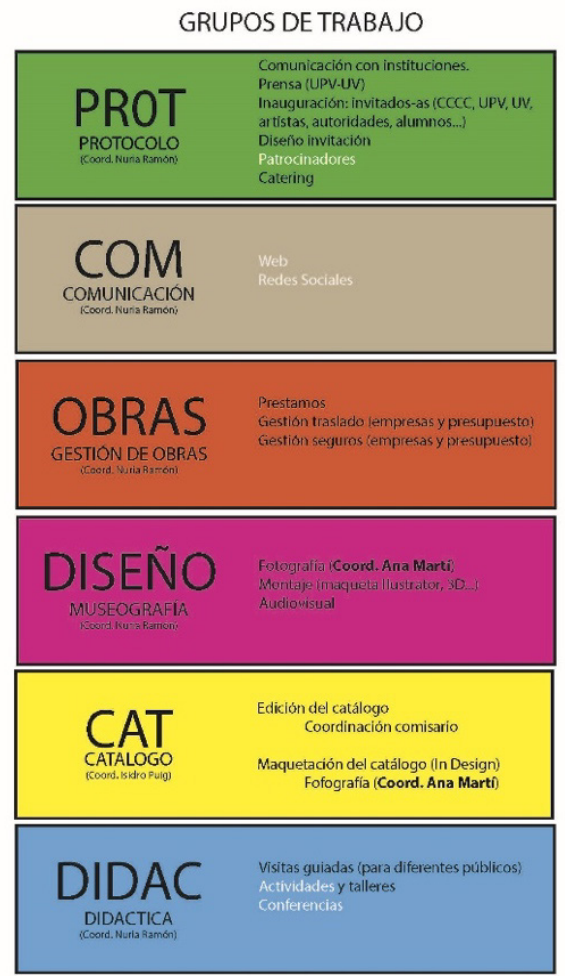

Fig. 1 Grupos de trabajo en los que se distribuye la actividad.

(cc) B Br-NC-ND 2020, Universitat Politècnica de València

Congreso In-Red (2020) 
Los alumnos y alumnas se debían de inscribir a cada una de estas áreas que habían sido diseñadas por el equipo docente en función del perfil o preferencias de cada uno de ellos/as. Debemos recordar como hemos indicado anteriormente que, al tratarse de un máster, el perfil del alumnado es muy variado. Así, los alumnos se distribuyeron en grupos de 6 estudiantes. Además, cada equipo debía nombrar un representante elegido de entre el resto de los componentes, quien se encargaba de centralizar la información que se iban generando.

Dada la estructura del proyecto en la distribución de estos grupos y, teniendo en cuenta que debían coordinarse conjuntamente más de 40 personas, se optó por que todos los estudiantes utilizaran la plataforma de Office 365 de Microsoft que proporciona la institución tanto al personal PDI como a los alumnos y alumnas. Esto nos permitía a todos los integrantes estar en continuo contacto, puesto que, se trataba de una actividad práctica cuyo periodo de ejecución iba a prolongarse a lo largo del segundo semestre. El primer paso fue conseguir que todo el alumnado tuviera acceso a la plataforma Office 365. En este sentido, el equipo docente tuvo que informar a los alumnos y alumnas matriculados a través de la Universitat de València para que se dieran de alta en la aplicación, ya que, al ser de otra universidad, necesitaban ser agregados por los profesores/as. Dentro de la plataforma de Office 365 existen diferentes aplicaciones y se decidió utilizar TEAMS para gestionar las distintas aplicaciones desde ésta. Se trata de una aplicación que favorece el trabajo en equipo y a distancia, donde se pueden editar documentos conjuntamente, realizar video conferencias, asignar tareas, organizar calendarios, etc... en resumen, lo que precisábamos para trabajar y coordinarnos una vez se hubiesen terminado las clases.

En la aplicación de TEAMS se creó un equipo general al que tenían acceso todos los participantes, donde se iba publicando todo el material que se generaba en el desarrollo general del proyecto, ya que era necesario que todos los grupos lo conocieran y tuvieran acceso para desempeñar correctamente su trabajo. Además, los/as estudiantes podían realizar las consultas de los aspectos frecuentes de la asignatura. A su vez se generaron una serie de subgrupos según cada grupo de trabajo, con el fin de descongestionar la carga de mensajes y que, las posibles dudas o preguntas correspondientes a cada sección se pudieran atender de una forma rápida y eficiente por parte del profesorado. No obstante, todos los alumnos/as y profesores/as teníamos acceso a cualquier grupo pudiendo en cualquier momento saber qué se estaba desarrollando en otros equipos. Además, se organizaron los documentos y recursos de cada grupo en función de las necesidades, de manera que en el equipo general se trabajó con un calendario global y, en cada subgrupo los alumnos y alumnas se coordinaron y organizaron sus propios calendarios y tareas.

Con el fin de poder comprobar la evolución del proyecto por cada grupo de manera presencial, se establecieron una serie de puntos de control a lo largo del semestre en los que cada equipo debía de poner en común con el resto de la clase los avances que se iban produciendo dentro de cada una de sus responsabilidades. Estos puntos de control, a su vez, permitían solucionar los posibles contratiempos a los que se enfrentaban los/as estudiantes y que no se habían podido resolver a través de TEAMS, A su vez permitía establecer un feed back con el resto del grupo y de los profesores. 
Como hemos indicado anteriormente, a través de la plataforma TEAMS eran supervisadas todas las acciones que realizaban los/las alumnos/as. Para ello, fue fundamental el uso de la herramienta Archivos en la que todos los documentos que se generaban debían subirse indicando, a través del chat, el título del documento que se había subido. Entre los archivos que se generaron en primer lugar fue la creación de un listado con las posibles obras de arte que eran susceptibles de ser expuestas y que proporcionaron los comisarios de la exposición. El hecho de que este documento estuviera en la aplicación permitía que los componentes del grupo lo pudieran editar conforme se iban actualizando los datos, tanto de localización de la obra, correcciones de título o disponibilidad de la pieza para la exposición. Pero, por otro lado, permitía a los profesores realizar correcciones y sugerencias ante posibles dudas, mientras que el resto de los compañeros de otros grupos podían consultar los listados para cualquier requisito que tuviesen por su parte.

A partir del Excel con las obras, lo primero que se hizo fue la localización y toma de fotografías de estas. Estas actividades eran trabajos de campo que, la mayor parte de las veces, iban acompañadas del profesor/a responsable de la actividad. Estas imágenes se subieron a una carpeta en el equipo General de la Exposición pues, eran imprescindibles para que el resto de los equipos participantes en el proyecto pudiera disponer de ellas y desarrollar su trabajo. 


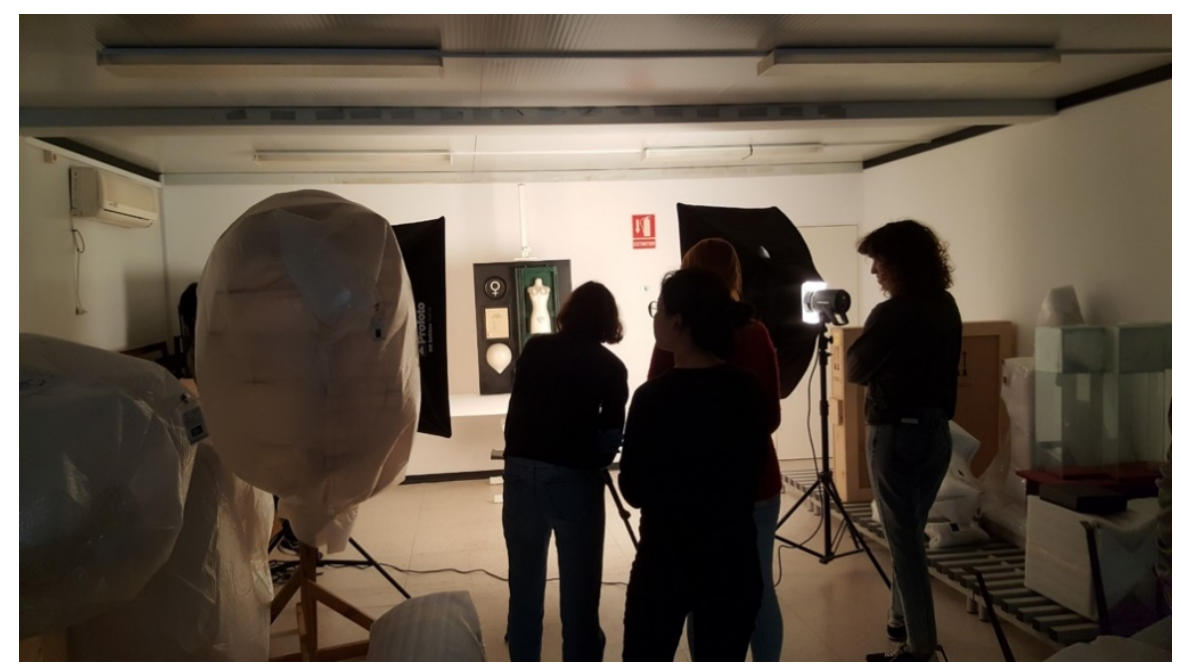

Fig. 3 Grupo de alumnas realizando las fotografias de las obras en los almacenes del Fondo de Arte de la UPV

Otro factor muy positivo de la aplicación de esta tipología de docencia fue que entre los equipos debía de existir una coordinación permanente, ya que, podía darse el caso de que un grupo realizara una tarea necesaria para que otro equipo pudiera avanzar en su trabajo. Un ejemplo de esta coordinación se observó en el contacto que se tuvo que realizar con la institución del Centre del Carme Cultura Contemporania en la que la primera gestión la realizó el grupo de Protocolo y, acto seguido, le pasó el testigo a los otros dos grupos implicados que eran Gestión de Obra y Diseño expositivo. Este último tuvo equipo tuvo que realizar una serie de trámites, como la solicitud de los planos, concretar la fecha exacta de la exposición y, la toma de medidas de la sala de exposiciones, ya que se tenían que comprobar "in situ" que las medidas de los planos se correspondían exactamente con las medidas de la sala.

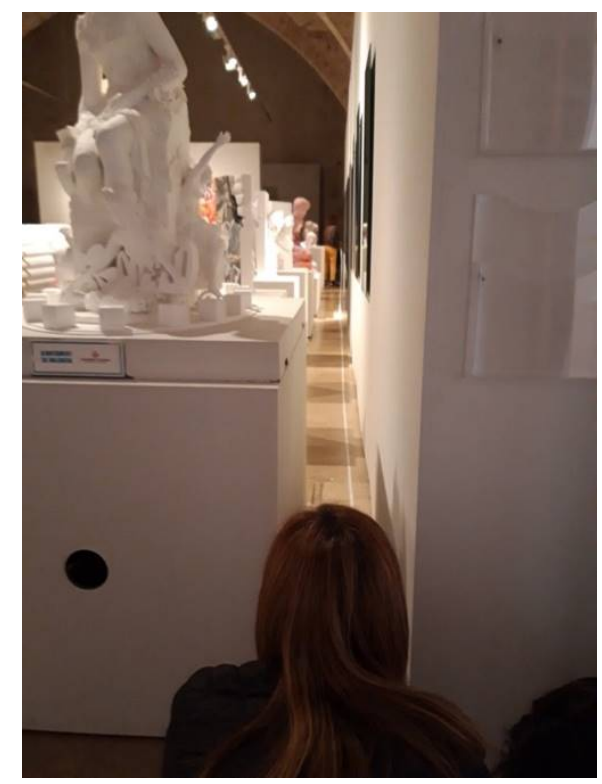

Fig. 4 Grupo de alumnos midiendo la sala de exposiciones del Centre del Carme 
Una vez comprobado el plano, se procedió al diseño de la exposición en la que los/as alumnos/as hicieron una primera propuesta que se puso en común en uno de los puntos de control y, en la que compartieron, con el resto de sus compañeros la necesidad de eliminar una parte de las obras al no caber todas en la sala de exposiciones.

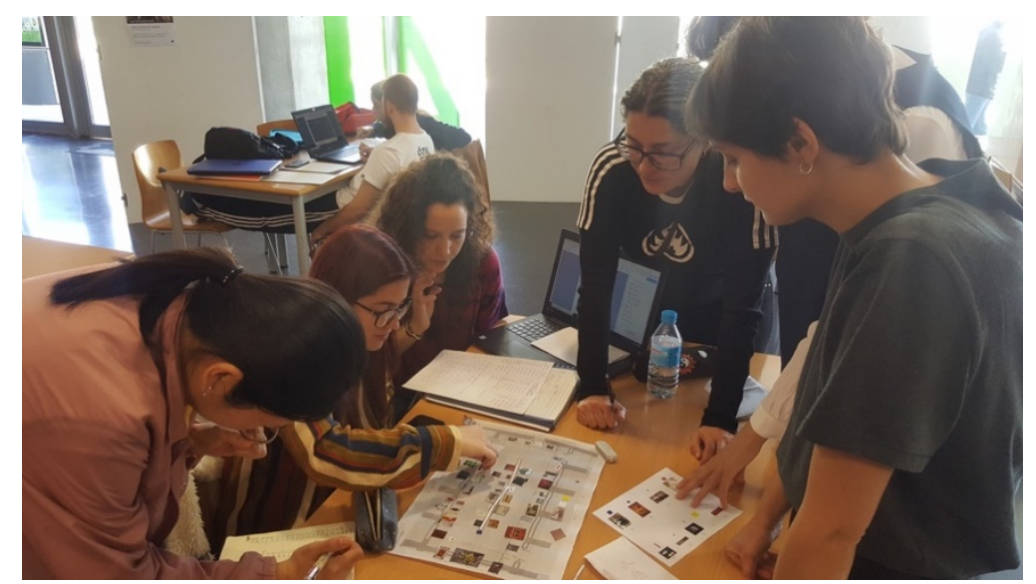

Fig. 5 Alumnas del grupo de Diseño expositivo distribuyendo la obra sobre plano

El trabajo cooperativo fue fundamental para el equipo docente, puesto que el profesional de la gestión cultural se ve obligado a colaborar con equipos interdisciplinares. En este sentido, los profesores instamos a los alumnos a reunirse en la Casa del Alumno puesto que era un espacio de la propia UPV en el que podían mantener reuniones sin ningún problema. Para constatar estas reuniones, los/as alumnos/as debían subir una fotografía, a la aplicación y en el apartado Archivos de su propio grupo, como testimonio de la acción.

Paralelamente al trabajo del diseño expositivo, tal y como hemos advertido al principio del desarrollo del proyecto, los equipos de Comunicación, Catálogo y Didáctica debían ir desarrollando sus propuestas.

Cabe decir, que todos los grupos trabajaban en función de una fecha crucial que era el día de inauguración de la exposición. En un principio la inauguración estaba programada para finales de mayo, coincidiendo con el final de la asignatura. El hecho de la inauguración de la exposición nos permitía al equipo docente la valoración de la actividad, así como, saber si los diferentes equipos habían cubierto sus objetivos, permitiéndonos realizar un acto de evaluación de forma más coherente y que nos ayudaba a ser conscientes de si habíamos sido capaces de que los alumnos y alumnas hubiesen alcanzado los objetivos según la guía docente de la asignatura. Sin embargo, a mediados de marzo nos indicaron desde el Centre del Carme Cultura Contemporania que debía retrasarse la fecha, como consecuencia de una reprogramación de actividades del propio centro, por lo que tuvo que posponerse la inauguración al mes de septiembre. Si bien este cambio de fechas supuso la necesidad de una reorganización, sobre todo en la manera en la que íbamos a evaluar esta actividad, también permitió que los estudiantes se enfrentaran a una de las características más comunes de la gestión cultural que consiste en los contratiempos surgidos en relación de la programación de actividades culturales. Ello favoreció a que los/las alumnos/as comprendieran de primera mano cómo podía afectar estas situaciones en su trabajo diario.

Por otro lado, este cambio obligó los profesores a mantener la fecha de mayo como la fecha límite de entrega del proyecto ya que la asignatura finalizaba y debía de ponerse la nota final en las actas durante el mes de junio. Para poder realizar el acto de evaluación correctamente, se cambió la metodología pasando 
de evaluar en lugar de la materialización de la exposición, a la presentación de los resultados a los que había llegado cada grupo de manera presencial frente al equipo docente y al resto de la clase. Este cambio en el procedimiento de evaluación fue positivo ya que los/as alumnos/as tuvieron la oportunidad de exponer las diversas situaciones a las que se habían enfrentado indicando cuál había sido la manera de proceder y, sobre todo, las conclusiones a las que habían llegado, que analizamos en el siguiente apartado del artículo. Además, junto con el acto de presentación del trabajo, debían de entregar una memoria de grupo que nos facilitaba a los profesores comprobar si habían seguido el procedimiento esperado.

Para analizar si la experiencia había sido positiva para el alumnado, establecimos un feed-back entre los alumnos/as y docentes. En este feed-back, que se realizó de forma grupal a partir de la pregunta ¿Crees que esta experiencia ha sido positiva para tu aprendizaje?, todos ellos consideraron como esta actividad altamente positiva y valorada para su aprendizaje y el futuro desarrollo de sus trabajos dentro de este campo.

\section{Resultados obtenidos}

La posibilidad de realizar y materializar una exposición en el Centro del Carme Cultura Contemporania, nos ha servido para poner en práctica diferentes metodologías apropiadas para los objetivos de la asignatura y la práctica de los principios de la nueva museología y el trabajo en grupo.

Al tener que relacionarse directamente con los agentes implicados en la organización de una exposición de tal envergadura, los/as alumnos/as aprendieron temas de protocolo, legislación, comunicación, etc. Además, desarrollaron habilidades a la hora de gestionar un archivo de arte, solicitar las obras, utilizando los documentos requeridos para todo ello.

Los equipos humanos multidisciplinares funcionaron excelentemente, logramos que se utilizara la plataforma de Office 365 eficazmente y, en concreto la aplicación de TEAMS, donde se volcaron todos los recursos. Ello nos permitió fluidez en la resolución de necesidades y conflictos. La oportunidad de poner en práctica el trabajo en equipo y, la coordinación de grupos grandes con muchas personas implicadas, facilitó que surgieran ideas innovadoras, y que los alumnos fueran conscientes de que tenían los conocimientos y las herramientas necesarias para enfrentarse a ello en el futuro. Esto se debe en gran medida a la utilización de TEAMS, junto a los puntos de control presenciales, que facilitaron que estuviésemos en contacto continuamente.

En las entrevistas realizadas a distintos estudiantes (un total de 8), y los comentarios recogidos en los puntos de control y la presentación final, se evidenció que en un principio la aplicación de TEAMS resultó compleja, puesto que los/as alumnos/as estaban acostumbrados a utilizar Google Drive, o incluso algunas plataformas sociales como WhatsApp o Facebook para comunicarse y compartir documentos. Pero todo fue cuestión de tiempo y, después de varias semanas, todos los alumnos y alumnas junto a los docentes nos pusimos de acuerdo en cómo utilizar la plataforma. Una vez superados estos problemas, reconocieron que fue de gran utilidad, e incluso, muy cómoda al poder instalarla en el teléfono móvil, recibir avisos cuando te nombraban, etc. No obstante, también debemos indicar que tanto algunos alumnos como algunos docentes terminaron saturados por la sobrecarga de información y la necesidad de estar conectados continuamente. Por ello, se optó por dedicar varias sesiones a explicar el funcionamiento de la plataforma y la configuración de los avisos y alertas, así como la planificación de acciones concretas y fechas de entrega en el calendario común, que resultó de gran ayuda. 
Además, los alumnos y alumnas aprendieron las necesidades reales de un proyecto expositivo, redactando un proyecto de gestión cultural que les pudiera servir para la práctica profesional futura, puesto que fueron conscientes de la necesidad de la investigación previa respecto del espacio y las obras, las necesidades del público, la sala, etc. Del mismo modo, aprendieron a programar los eventos, gestionar el tiempo, los recursos y las tareas relacionadas con el montaje de una exposición. De hecho, así lo confirmaron en las entrevistas. Muchos de los alumnos agradecieron la oportunidad de poner en práctica distintas cuestiones estudiadas en otras asignaturas y que, aunque se habían realizado prácticas de aula, no tenía comparación con la puesta en práctica real. Uno de los problemas más comunes fue la resolución de conflictos entre compañeros, de un mismo grupo y de distintitos grupos entre sí. Se evidenció que al llevar a la práctica un caso real surgen una serie de conflictos que deben ser subsanados con urgencia. La toma de decisiones y la capacidad de delegar y compartir tareas se vuelve crucial, y esto es muy complicado de emular o reproducir en el caso de la práctica de aula. Al tratarse de una práctica, fue importante remarcar el hecho de que se estaba aprendiendo, dejando la oportunidad a equivocarse, siempre con el soporte de los docentes, para que los errores no supusieran un problema. En este sentido, se evidenció que existía una importante carencia en el trabajo en equipo y colaborativo. Para tratar de subsanarlo, se realizaron distintas reuniones, y se aprovecharon las experiencias negativas para extraer conclusiones y aprendizajes significativos.

Por otro lado, la oportunidad de trabajar con auténticas obras de arte de artistas renombrados motivó a los alumnos y alumnas, a la vez que conocieron como trabaja el Fondo de Arte de la universidad en la que estudian, y la importancia de su patrimonio. También fue muy motivador trabajar con una institución tan importante como el Centre del Carme, conociendo el trasfondo de estas instituciones, su personal, etc. De nuevo surgieron algunos problemas en lo que se refiere a las formas y protocolos. En numerosas ocasiones se tuvo que revisar y corregir el trabajo realizado por los alumnos para comunicarse con terceros, y se solicitó que un docente debía revisar todas ellas antes de enviarlas. En distintas ocasiones se envió el mismo mensaje desde distintos medios y grupos, con el riego de saturar a los profesionales que colaboraban con el proyecto.

No obstante, los medios de comunicación utilizados en la exposición fueron adecuados, los alumnos y alumnas aprendieron cuales son más eficaces, y los necesarios, contactaron con la prensa, utilizando distintos canales en las redes sociales, redactando comunicados, etc. que les ayudó a conocer de primera mano las necesidades comunicativas. De hecho, la exposición fue muy bien valorada, tuvo un total de 6.588 visitantes según los datos facilitados por la propia institución, y fue ampliamente difundida en los medios de comunicación. Las actividades paralelas realizadas siguiendo los principios de la nueva museología, y el museo participativo, tuvieron bastante éxito. Se programaron algunas actividades críticas e inclusivas como los talleres para niños y las actividades paralelas.

\section{Conclusiones}

El reto planteado a la hora de organizar esta exposición como una actividad docente en la que implicar a todo el alumnado de una asignatura del máster de gestión cultural, supuso un gran trabajo añadido, pero permitió reforzar y enriquecer el proceso de enseñanza aprendizaje de una manera practica y eficiente, tanto para los docentes como para el alumnado. Pese a todos los problemas que fueron surgiendo en el desarrollo del ejercicio, estamos muy satisfechos con los resultados obtenidos en cuanto a la evaluación del aprendizaje de la experiencia. 
Por un lado, nos hemos tenido de enfrentar a los problemas derivados de la saturación, o la carga de trabajo en los alumnos, puesto que algunos asumieron más responsabilidades de las que les tocaban, y otros no llegaban a cumplir con las suyas. Para ello se evidenció la eficacia de los puntos de control, así como la revisión constante del trabajo del alumnado. No obstante, el hecho de estar inmersos en una actividad de tal envergadura benefició los aprendizajes significativos permitiéndonos aplicar todos los conocimientos estudiados en un caso práctico, real y motivador.

Por otro lado, debemos reconocer que en un principio tuvimos algunos problemas técnicos al utilizar la plataforma de TEAMS de Microsoft, pero finalmente la mezcla de clases presenciales y coordinación online junto a los puntos de control resultó muy muy eficaz para la organización de los grupos y solucionar los problemas que fueron surgiendo a tiempo. Estamos convencidos de que en un escenario como el actual, la formación del alumnado en el uso de las TIC resulta fundamental, sobre todo en lo que se refiere al trabajo colaborativo y a distancia.

Por lo general, las metodologías docentes utilizadas fueron las adecuadas, y logramos adaptarlas al caso de la exposición. Por ello, estamos convencidos de que estas metodologías pueden ser utilizadas por otros equipos curatoriales, u experiencias expositivas. La metodología del aprendizaje basado en problemas resultó de gran ayuda al afrontar el caso de la exposición, se intentó que los grupos no fuesen demasiado grandes y se comprobó cómo la metodología nos sirvió para mejorar el trabajo autónomo y creativo, puesto que fueron los propios alumnos quienes se iban encontrando con los problemas y acudían a los docentes cuando requerían ayuda. La metodología del Aprendizaje - Servicio motivó a los alumnos, y sirvió para que fuesen conscientes de la importancia de las dos instituciones públicas con las que trabajamos. Además, el Aprendizaje Cooperativo nos sirvió para que los distintos grupos se organizaran con las tareas asignadas a cada uno. Por medio de los problemas y situaciones a las que llegamos logramos evidenciar las necesidades reales, y el alumnado pudo ser consciente de la importancia del buen funcionamiento colaborativo en la vida profesional.

El comisariado y organización de exposiciones suele ser una práctica individual cuando se trabaja desde la museología tradicional, pero la nueva museología participativa nos ofrece la posibilidad de acercarnos a las audiencias de una manera diferente a la vez que hemos comprobado como el trabajo en equipo para este tipo de acciones puede otorgarnos un valor añadido.

El hecho de embarcarnos en un proyecto de tal envergadura motivó a los alumnos hacia la profesión del gestor cultural, viendo las implicaciones reales que tiene la profesión. La puesta en marcha de proyectos reales y plurales que impliquen a distintas instituciones ayuda a que el alumnado adquiera una práctica mucho más global, en la que intervienen una gran variedad de actores, desde administraciones públicas hasta entidades privadas. Resulta, además, muy gratificante cuando se materializa, los alumnos y alumnas se sienten realizados, y aprenden de lo que puede ser en el futuro la práctica profesional. Al implicar otras instituciones como el propio Fondo de arte de la universidad, o el Centro del Carmen, nos ha brindado la oportunidad de materializarlo en un proyecto real, lo que consideramos muy importante a la hora de adquirir las competencias necesarias en el futuro profesional de los gestores culturales. 


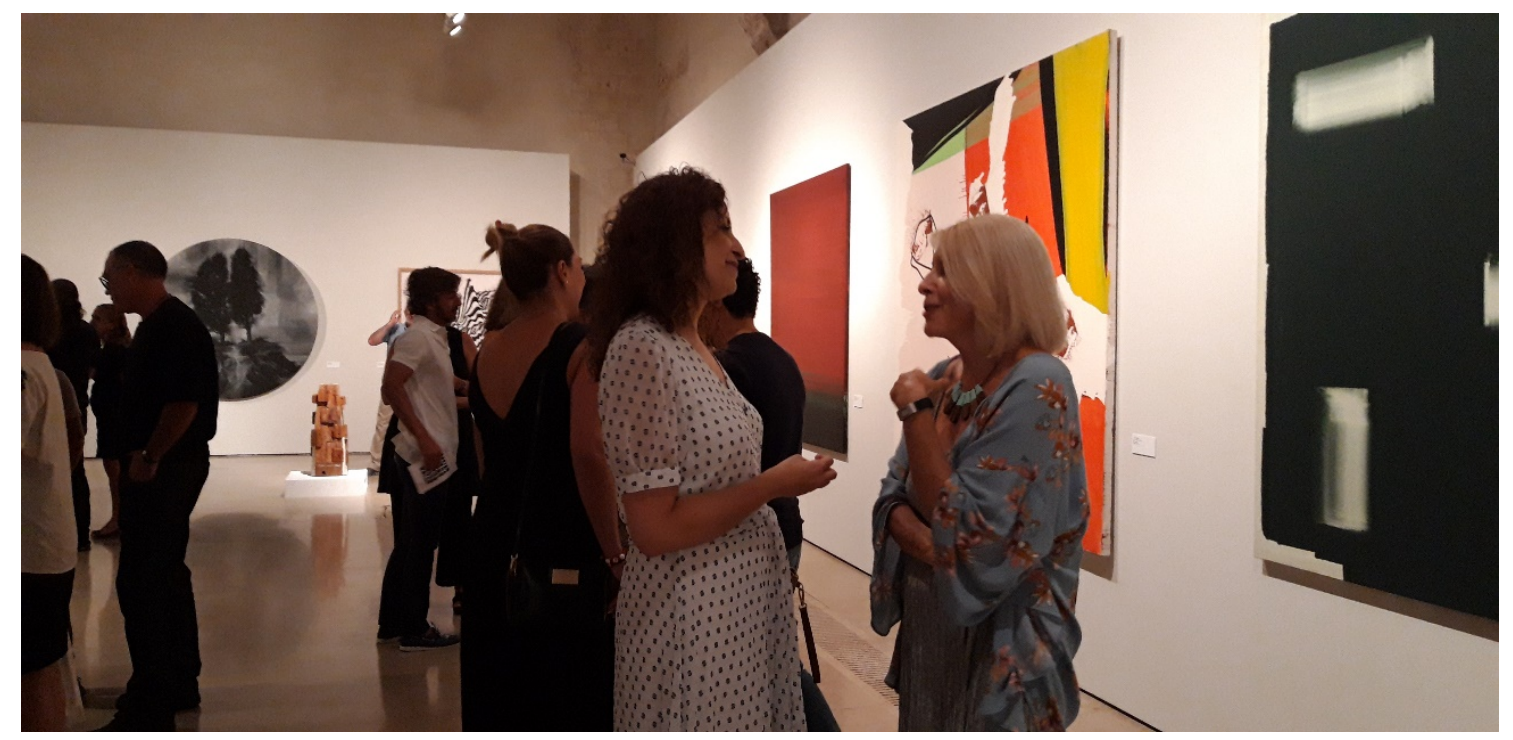

Fig. 6 Inauguración de la exposición en el Centro del Carmen el 6 de septiembre 2019

\section{Referencias}

BARROWS, H. (1986). A Taxonomy of problem-based learning methods. Medical Education(20), 481-486.

CABAÑÉS MARTINEZ, F. (2017). La profesión de gestor cultural. Apuntes sobre la situación actual. Culturas. Revista de Gestión Cultural. doi: DOI:https://doi.org/10.4995/cs.2017.7474

COCHRAN, J. (2017). The Skillful Curator: A Case Study in Curatorial Pedagogy and Collective ExhibitionMaking. Art History Pedagogy \& Practice. http://arthistoryteachingresources.org/2017/03/the-skillful-curator-a-casestudy-in-curatorial-pedagogy-and-collective-exhibition-making/ [Consulta: 17 de marzo de 2020].

DÍAZ, M. D. (2005). Modalidades de Enseñanza Centradas en el Desarrollo de Competencias. Orientaciones para Promover el Cambio Metodológico en el Espacio Europeo de Educación Superior. Ediciones Universidad de Oviedo. https://www2.ulpgc.es/hege/almacen/download/42/42376/modalidades_ensenanza_competencias_mario_miguel2 do cumento.pdf [Consulta: 12 de octubre de 2019].

FRANCISCO, A., \& MOLINER, L. (2010). El Aprendizaje Servicio en la Universidad: una estrategia en la formación de ciudadanía crítica. Reifop, 13(4), 69-77.

KRZYS ACORD, S. (2010). Beyond the Head: The Practical Work of Curating Contemporary Art. Qualitative Sociology volume, 33, 447-467.

LA ASOCIACIÓN ESPAÑOLA DE GESTORES DE PATRIMONIO CULTURAL. (s.f). EL GESTOR CULTURAL. http://aegpc.org/gestion-y-gestor-cultural\#elgestor. [Consulta: 17 de marzo de 2020].

MARTÍNEZ-ODRÍA, A. (2007). Service-Learning o Aprendizaje-Servicio. La Apertura de la Escuela a la Comunidad Local como Propuesta de Educación para la Ciudadanía. Bordon, 59(4), 627-640.

JOHNSON, R., JOHNSON D.W. (1994). An Overview of Cooperative Learning. http://digsys.upc.es/ed/general/Gasteiz/docs_ac/Johnson_Overview_of_Cooperative_Learning.pdf

SCHONFELD, R., \& WESTERMANN, M. (2015). The Andrew W. Mellon Foundation Art Museum Staff Demographic Survey. Andrew W. Mellon Foundation. https://mellon.org/media/filer_public/ba/99/ba99e53a-48d54038-80e1-66f9ba1c020e/awmf_museum_diversity_report_aamd_7-28-15.pdf

SULLIVAN, E. J. (s.f). Curating as a Teaching and Learning Process. https://www.nyucuratorialcollaborative.org/about/ [Consulta: 17 de marzo de 2020].

VILLENEUVE, P. (2019). Considering Competing Values in Art Museum Exhibition Curation. Stedelijk Studies. 